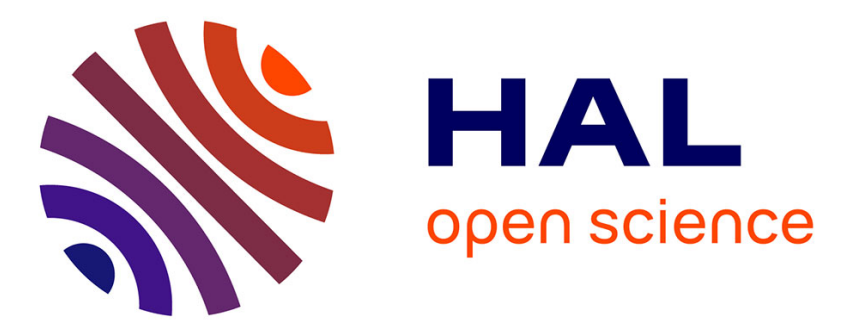

\title{
Generalized de Bruijn Words and the State Complexity of Conjugate Sets
}

Daniel Gabric, Štěpán Holub, Jeffrey Shallit

\section{To cite this version:}

Daniel Gabric, Štěpán Holub, Jeffrey Shallit. Generalized de Bruijn Words and the State Complexity of Conjugate Sets. 21th International Conference on Descriptional Complexity of Formal Systems (DCFS), Jul 2019, Košice, Slovakia. pp.137-146, 10.1007/978-3-030-23247-4_10 . hal-02387301

\section{HAL Id: hal-02387301 \\ https://hal.inria.fr/hal-02387301}

Submitted on 29 Nov 2019

HAL is a multi-disciplinary open access archive for the deposit and dissemination of scientific research documents, whether they are published or not. The documents may come from teaching and research institutions in France or abroad, or from public or private research centers.
L'archive ouverte pluridisciplinaire HAL, est destinée au dépôt et à la diffusion de documents scientifiques de niveau recherche, publiés ou non, émanant des établissements d'enseignement et de recherche français ou étrangers, des laboratoires publics ou privés.

\section{(c)(1)}

Distributed under a Creative Commons Attribution| 4.0 International License 


\title{
Generalized de Bruijn words and the state complexity of conjugate sets
}

\author{
Daniel Gabric ${ }^{1}$, Štěpán Holub ${ }^{2}$, and Jeffrey Shallit ${ }^{1}$ \\ 1 School of Computer Science \\ University of Waterloo \\ Waterloo, Ontario N2L 3G1 \\ Canada \\ \{dgabric, shallit\}@uwaterloo.ca \\ 2 Department of Algebra \\ Faculty of Mathematics and Physics \\ Charles University \\ Prague \\ Czech Republic \\ holub@karlin.mff.cuni.cz
}

\begin{abstract}
We consider a certain natural generalization of de Bruijn words, and use it to compute the exact maximum state complexity for the language consisting of the conjugates of a single word. In other words, we determine the state complexity of cyclic shift on languages consisting of a single word.
\end{abstract}

\section{Introduction}

Let $x, y$ be words. We say $x$ and $y$ are conjugates if one is a cyclic shift of the other; equivalently, if there exist words $u, v$ such that $x=u v$ and $y=v u$. For example, the English words listen and enlist are conjugates.

The set of all conjugates of a word $x$ is denoted by $C(x)$. Thus, for example, $C$ (eat) $=\{$ eat, tea, ate $\}$. We also write $C(L)$ for the set of all conjugates of elements of the language $L$.

For a regular language $L$ let $\operatorname{sc}(L)$ denote the state complexity of $L$ : the number of states in the smallest complete DFA accepting $L$. State complexity is sometimes also called quotient complexity [5]. The state complexity of the cyclic shift operation $L \rightarrow C(L)$ for arbitrary regular languages $L$ was studied in Maslov's pioneering 1970 paper [17]. More recently, Jirásková and Okhotin [14] improved Maslov's bound, and Jirásek and Jirásková studied the state complexity of the conjugates of prefix-free languages [13].

In this note we investigate the state complexity of the finite language $C(x)$, over all words $x$ of length $N$. In other words, we determine the state complexity of cyclic shift on languages consisting of a single word. Clearly $\operatorname{sc}(C(x))$ achieves its minimum - namely, $N+2$ - at words of the form $a^{N}$, where $a$ is a single letter. By considering random words, it seems likely that $\operatorname{sc}(C(x))=O\left(N^{2}\right)$.

Our main result makes this precise: 
Theorem 1. Let $\Sigma_{k}$ be an alphabet of cardinality $k \geq 2$, and let $N \geq 1$ be an integer. Define $r=\left\lfloor\log _{k} N\right\rfloor$ and $v=\left(k^{r+1}-1\right) /(k-1)$. Then

$$
\max _{w \in \Sigma_{k}^{n}} \operatorname{sc}(C(w))=2 v+N(N-2 r-1)+1 .
$$

Furthermore, we characterize those words $x$ achieving this maximum.

Our theorem depends on a certain natural generalization of de Bruijn words, of independent interest, which is introduced in the next section.

\section{Generalized de Bruijn words}

De Bruijn words (also called de Bruijn sequences) have a long history $[8,16,10,3,4]$, and have been extremely well studied $[9,18]$. Let $\Sigma_{k}$ denote the $k$-letter alphabet $\{0,1, \ldots, k-1\}$. Traditionally, there are two distinct ways of thinking about these words: for integers $k \geq 2, n \geq 1$ they are

(a) the words $w$ having each word of length $n$ over $\Sigma_{k}$ exactly once as a factor; or

(b) the words $w$ having each word of length $n$ over $\Sigma_{k}$ exactly once as a factor, when $w$ is considered as a "circular word", or "necklace", where the word "wraps around" at the end back to the beginning.

For example, for $k=2$ and $n=4$, the word

$$
0000111101100101000
$$

is an example of the first interpretation and

$$
0000111101100101
$$

is an example of the second.

In this paper, we are concerned with the second (circular) interpretation of de Bruijn words, and we write $D(k, n)$ for the set of all such words. Obviously, such words exist only for lengths of the form $k^{n}$. Is there a sensible way to generalize this class of words so that one could speak fruitfully of (generalized) de Bruijn words of every length?

One natural way to do so is to use the notion of subword complexity (also called factor complexity or just complexity). For $0 \leq i \leq N$ let $\gamma_{i}(w)$ denote the number of distinct length- $i$ factors of the word $w \in \Sigma_{k}^{N}$ (considered circularly). For all words $w$, there is a natural upper bound on $\gamma_{i}(w)$ for $0 \leq i \leq N$, as follows:

$$
\gamma_{i}(w) \leq \min \left(k^{i}, N\right) .
$$

This is immediate, since there are at most $k^{i}$ words of length $i$ over $\Sigma_{k}$, and there are at most $N$ positions where a word could begin in $w$ (considered circularly). 
Ordinary de Bruijn words are then precisely those words $w$ of length $k^{n}$ for which $\gamma_{n}(w)=k^{n}$. But even more is true: $w \in D(k, n)$ also achieves the upper bound in (1) for all $i \leq k^{n}$. To see this, note that if $i \leq n$, then every word of length $i$ occurs as a prefix of some word of length $n$, and every word of length $n$ is guaranteed to appear in $w$. On the other hand, all $k^{n}$ (circular) factors of each length $i \geq n$ are distinct, because their length- $n$ prefixes are all distinct.

This motivates the following definition:

Definition 1. A word $x$ of length $N$ over a k-letter alphabet is said to be a generalized de Bruijn word if $\gamma_{i}(x)=\min \left(k^{i}, N\right)$ for all $0 \leq i \leq N$.

Table 1 gives the lexicographically least de Bruijn words for a two-letter alphabet, for lengths 1 to 31, and the number of such words (counted up to cyclic shift). This forms sequence $\underline{A} 317586$ in the On-Line Encyclopedia of Integer Sequences (OEIS) [20]. The second author has computed these numbers up to $N=63$. words.

We point out an alternative characterization of our generalized de Bruijn

Proposition 1. A word $w \in \Sigma_{k}^{N}$ is a generalized de Bruijn word iff both of the following hold:

(a) $\gamma_{r}(w)=k^{r}$; and

(b) $\gamma_{r+1}(w)=N$,

where $r=\left\lfloor\log _{k} N\right\rfloor$.

Proof. A generalized de Bruijn word trivially has these properties. An argument similar to the discussion before Definition 1 shows that the two properties imply the bound in Eq. (1).

The main result of this section is the following.

Theorem 2. For all integers $k \geq 2$ and $N \geq 1$ there exists a generalized de Bruijn word of length $N$ over a k-letter alphabet.

Proof. For $k=2$ the proof can be found in [19], although strangely it is not explicitly stated anywhere in the paper. (Lemma 3 implies it.)

For $k>2$ we can derive this result from a paper by Lempel [15]. Lempel proved that for all $k \geq 2, n \geq 1, N \leq k^{n}$, there exists a circular word $w=$ $w(k, n, N)$ of length $N$ for which the factors of size $n$ are distinct. (Also see $[11,6]$.) However, as stated, this result is not strong enough for our purposes. For example, there are circular words, such as 000101 of length 6 , having 6 distinct factors of length 4 , but only 3 distinct factors of length 2 . For our purposes, then, we need a stronger version of the result, which can nevertheless be obtained from a further analysis of Lempel's proof. 


\begin{tabular}{|c|c|c|}
\hline & $\begin{array}{l}\text { lexicographically least generalized } \\
\text { binary de Bruijn word of length } N\end{array}$ & $\begin{array}{l}\text { number of } \\
\text { such words }\end{array}$ \\
\hline 1 & 0 & 2 \\
\hline 2 & 01 & 1 \\
\hline 3 & 001 & 2 \\
\hline 4 & 0011 & 1 \\
\hline 5 & 00011 & 2 \\
\hline 6 & 000111 & 3 \\
\hline 7 & 0001011 & 4 \\
\hline 8 & 00010111 & 2 \\
\hline 9 & 000010111 & 4 \\
\hline 10 & 0000101111 & 3 \\
\hline 11 & 00001011101 & 6 \\
\hline 12 & 000010100111 & 13 \\
\hline 13 & 0000100110111 & 12 \\
\hline 14 & 00001001101111 & 20 \\
\hline 15 & 000010011010111 & 32 \\
\hline 16 & 0000100110101111 & 16 \\
\hline 17 & 00000100110101111 & 32 \\
\hline 18 & 000001001101011111 & 36 \\
\hline 19 & 0000010100110101111 & 68 \\
\hline 20 & 00000100101100111101 & 141 \\
\hline 21 & 000001000110100101111 & 242 \\
\hline 22 & 0000010001101001011111 & 407 \\
\hline 23 & 00000100011001110101111 & 600 \\
\hline 24 & 000001000110010101101111 & 898 \\
\hline 25 & 0000010001100101011011111 & 1440 \\
\hline 26 & 00000100011001010011101111 & 1812 \\
\hline 27 & 000001000110010100111011111 & 2000 \\
\hline 28 & 0000010001100101001110101111 & 2480 \\
\hline 29 & 00000100011001010011101011111 & 2176 \\
\hline 30 & 000001000110010110100111011111 & 2816 \\
\hline $31 \mid$ & |0000010001100101001110101101111 & 4096 \\
\hline
\end{tabular}

Table 1. Generalized de Bruijn words

An Eulerian graph is a directed graph in which, for each vertex $v$, the indegree of $v$ is equal to the outdegree of $v$. By a closed chain we mean a sequence of edges $\left(a, v_{1}\right),\left(v_{1}, v_{2}\right),\left(v_{2}, v_{3}\right), \ldots,\left(v_{n-1}, a\right)$, where each edge is distinct, but vertices may be repeated. Each closed chain forms an Eulerian graph and each connected Eulerian graph admits a closed chain containing all its edges.

Let $G_{k}^{n}$ be the $k$-ary de Bruijn graph of order $n$. This is a directed graph where the vertices are the words of length $n$, and edges join a word $x$ to a word $y$ if $x=a t$ and $y=t b$ for some letters $a, b$ and a word $t$. So every vertex of $G_{k}^{n}$ has $k$ incoming edges, and $k$ outgoing edges, and therefore the underlying graph $G_{k}^{n}$ is regular of degree $2 k$. By Proposition 1, building a generalized de Bruijn word of length $N=k^{n}+j$, where $0 \leq j \leq(k-1) k^{n}$, over a $k$-letter alphabet 
then amounts to constructing a closed chain of length $N$ in $G_{k}^{n}$ that visits every vertex.

One of Lempel's main results [15, Theorem 1] states that such a closed chain exists, but does not mention explicitly whether it visits every vertex. In the proof, the chain is obtained by constructing a connected Eulerian graph using $[15$, Lemma 6$]$. Now, the analysis of the proof of [15, Lemma 6] shows that the constructed Eulerian graph is not only connected (which is the explicit concern of the lemma) but also spanning. The closed chain is eventually obtained as a complement of a graph $G$ (denoted as $T_{p}$ in [15]), where $G$ is an Eulerian graph contained in $G_{k}^{n}$ such that the degree of each vertex in $G$ is at most $2(k-1)$. Therefore, its complement is obviously spanning.

Remark 1. We have not been able to find this precise notion of generalized de Bruijn word in the literature anywhere, although there are some papers that come very close. For example, Iványi [12] considered the analogue of Eq. (1) for ordinary (non-circular) words. He called a word $w$ supercomplex if the analogue of the upper bound (1) is attained not only for $w$, but also for all prefixes of $w$. However, binary supercomplex words do not exist past length 9 . The third author also considered the analogue of Eq. (1) for ordinary words [19]. However, Lemma 3 of that paper actually implies the existence of our generalized (circular) de Bruijn words of every length over a binary alphabet, although this was not stated explicitly. Anisiu, Blázsik, and Kása [2] discussed a related concept: namely, those length- $N$ words $w$ for which $\max _{1 \leq i \leq N} \rho_{i}(w)=\max _{x \in \Sigma_{k}^{N}} \max _{1 \leq i \leq N} \rho_{i}(x)$ where $\rho_{i}(w)$ denotes the number of distinct length- $i$ factors of $w$ (here considered in the ordinary sense, not circularly). Also see [7].

We now count the total number of factors of a generalized de Bruijn word. This is a generalization of Theorem 2 of [19] to all $k \geq 2$, adapted for the case of circular words.

Proposition 2. If $w \in \Sigma_{k}^{N}$ is a generalized de Bruijn word, then

$$
\sum_{0 \leq i \leq N} \gamma_{i}(w)=\frac{k^{r+1}-1}{k-1}+N(N-r),
$$

where $r=\left\lfloor\log _{k} N\right\rfloor$.

Proof. We have

$$
\begin{aligned}
\sum_{0 \leq i \leq N} \gamma_{i}(w) & =\sum_{0 \leq i \leq N} \min \left(k^{i}, N\right) \\
& =\sum_{0 \leq i \leq r} k^{i}+\sum_{r<i \leq N} N \\
& =\frac{k^{r+1}-1}{k-1}+N(N-r) .
\end{aligned}
$$




\section{State complexity}

We start with a general upper bound on state complexity.

Theorem 3. Let $\Sigma$ be an alphabet of cardinality $k \geq 2$ and let $L \subseteq \Sigma^{N}$. Define $m=|L|, r=\left\lfloor\log _{k} m\right\rfloor$ and $v=\left(k^{r+1}-1\right) /(k-1)$. If $N \geq 2 r+1$ then $\operatorname{sc}(L) \leq$ $2 v+m(N-2 r-1)+1$.

Proof. A level is a set of all nodes at a particular distance from the root. The complete $k$-ary tree of $r+1$ levels therefore corresponds to words of length $\leq r$, and the total number of nodes in this tree is $1+k+\cdots+k^{r}=\frac{k^{r+1}-1}{k-1}$.

The language $L$ can be accepted by a DFA with the following topology: there is a complete $k$-ary tree of $r+1$ levels rooted at the initial state $p_{\epsilon}$. At the very next level there are at most $m$ nodes, and these nodes form the roots of at most $m$ chains of $N-2 r-1$ nodes each. These chains need not be disjoint, but will be in the worst case. At the end, there is another complete $k$-ary tree of $r+1$ levels culminating in a single accepting state. Finally, there is also a single nonaccepting state that captures all transitions not yet defined. The total number of states is therefore $2 v+m(N-2 r-1)+1$.

More formally, define

$$
\begin{aligned}
& X=\Sigma^{\leq r} \cup\{x: r<|x|<N-r-1 \text { and } x \text { is a prefix of an element of } L\} \\
& Y=\{y:|y|=N-r-1 \text { and } y \text { is a prefix of an element of } L\}
\end{aligned}
$$

The states of our DFA are $d$, a "dead" state; $p_{x}$, for $x \in X$; and $s_{z}$, for all $z$ with $|z| \leq r$. The states $p_{x}$ correspond to prefixes of words of $L$ and the states $s_{z}$ correspond to suffixes of words of $L$.

The initial state is $p_{\epsilon}$.

The transitions are given by $\delta\left(p_{x}, a\right)=p_{x a}$ for $x \in X$ and $a \in \Sigma$ and $\delta\left(p_{y}, a\right)=s_{z}$, if $y \in Y$ and $y a z \in L ; \delta\left(s_{a v}, a\right)=s_{v}$ for $v \in \Sigma^{<i}$ and $a \in \Sigma$. All other transitions go to $d$.

Finally, the unique final state is $s_{\epsilon}$.

This construction is illustrated in Figure 1 for $k=2, N=12, m=10, r=3$, $v=15, N-2 r-1=5$, and

$$
\begin{aligned}
& L=\{000010100000,000101100010,011110100001,100110011111,101011110111, \\
& 110100100110,110101010011,110110101101,111001100101,111110110100\} .
\end{aligned}
$$




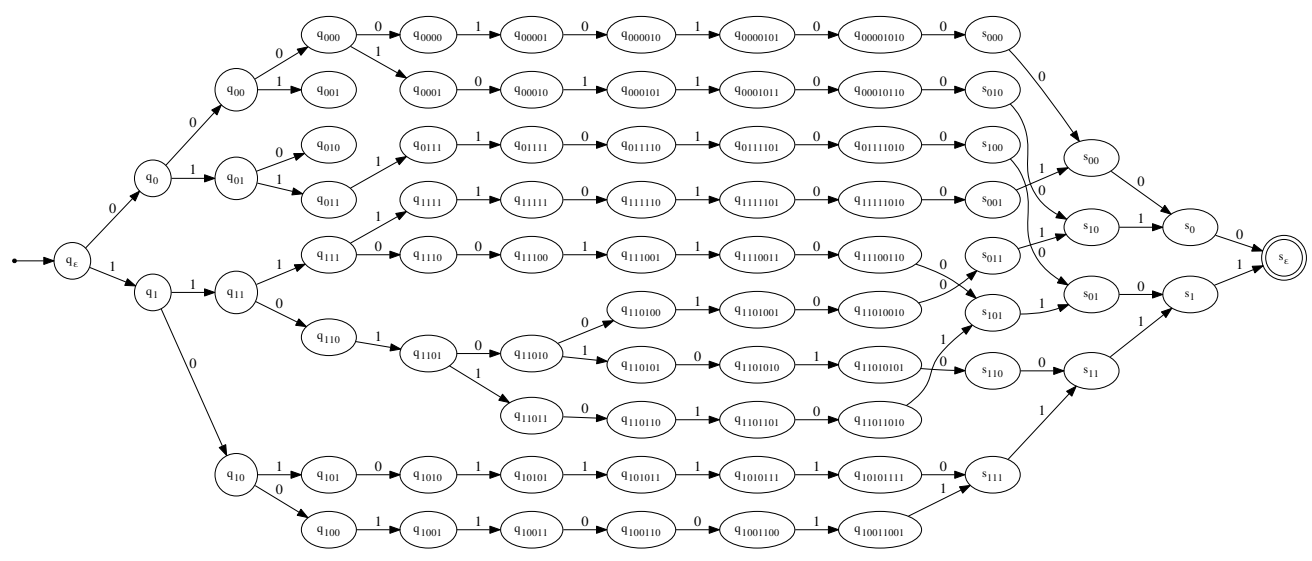

Fig. 1. Example of the construction

As a corollary, we now get an upper bound on $\operatorname{sc}(C(x))$ :

Corollary 1. If $x$ is a word of length $N$ over a $k$-letter alphabet, with $k \geq 2$, then

$$
\operatorname{sc}(C(x)) \leq 2 v+N(N-2 r-1)+1,
$$

where $r=\left\lfloor\log _{k} n\right\rfloor$ and $v=\left(k^{r+1}-1\right) /(k-1)$.

Proof. Let $x$ be a word of length $N$, and let $L=C(x)$. Set $m=|L| \leq N$, $r=\left\lfloor\log _{k} N\right\rfloor$ and $v=\left(k^{r+1}-1\right) /(k-1)$. The inequality $N \geq 2 r+1$ holds in all cases except $k=2$ and $n=2$; this case can be checked separately. Theorem 3 therefore yields $\operatorname{sc}(L) \leq 2 v+N(N-2 r-1)+1$, as desired.

It now remains to prove that there exist words that achieve this upper bound. In fact, such words are exactly the generalized de Bruijn words defined in Section 2 .

Theorem 4. A length- $N$ word $x$ over a $k$-letter alphabet satisfies

$$
\operatorname{sc}(C(x))=2 v+N(N-2 r-1)+1,
$$

where $r=\left\lfloor\log _{k} N\right\rfloor$ and $v=\left(k^{r+1}-1\right) /(k-1)$ iff $x$ is a generalized de Bruijn word.

Proof. Suppose $x$ is a generalized de Bruijn word. We first show that there are $2 v+N(N-2 r-1)+1$ inequivalent words for the Myhill-Nerode equivalence relation $R$ associated with $C(x)$. This will show $\operatorname{sc}(C(x)) \geq 2 v+N(N-2 r-1)+1$ and hence, by Corollary 1 , that $\operatorname{sc}(C(x))=2 v+N(N-2 r-1)+1$. 
Representatives of the Myhill-Nerode classes can be classified as follows:

(a) all the words of length $\leq r$;

(b) all the factors of conjugates of $x$ of length $\ell$, for $r<\ell<N-r$;

(c) for each word $w$ of length $\leq r$, the lexicographically least factor $z$ of $C(x)$ of length $N-r$ for which $z w \in C(x)$.

(d) the single equivalence class corresponding to words not in $C(x)$.

There are $v$ words in (a), and $v$ words in (c), there are $N(N-2 r-1)$ words in (b), and one word in (d).

We need to see that these are all inequivalent. Since all the words in $C(x)$ are of length $N$, no two factors of different lengths can be equivalent. It therefore suffices to examine pairs of words of identical length.

In group (a), let $y, z$ be two distinct words of length $j \leq r$. Since $x$, considered circularly, contains all factors of length $r=\left\lfloor\log _{k} N\right\rfloor$, it contains $y$ and $z$ as factors. Let $y y^{\prime}$ (resp., $z z^{\prime}$ ) be a conjugate of $x$ with prefix $y$ (resp., $z$ ). Then $\left|y^{\prime}\right|=\left|z^{\prime}\right|=N-j \geq r+1$. If both $y z^{\prime}$ and $z z^{\prime}$ occur in $C(x)$, we would have two separate occurrences of $z^{\prime}$ in $x$ (considered circularly), which is impossible since $x$ is of length $N$ and has $N$ distinct factors of length $N-j$ (considered circularly). So $y z^{\prime} \notin C(x)$ and $y, z$ are inequivalent under Myhill-Nerode. This gives $v=\left(k^{r+1}-1\right) /(k-1)$ equivalence classes.

In group (b), let $y, z$ be two distinct factors of $C(x)$ (considered circularly) of length $j$ with $r<j<N-r$. Since $x$ is of length $N$ and contains $N$ distinct factors of length $r$, the first $r$ symbols of $y$ (resp., $z$ ) uniquely determine the position of $y$ (resp., $z$ ) within $x$ (considered as a circular word). So there is a unique $y^{\prime}$ such that $y y^{\prime} \in C(x)$, and similarly, there is a unique $z^{\prime}$ such that $z z^{\prime} \in C(x)$. Just as in case (a), since $\left|y^{\prime}\right|=\left|z^{\prime}\right| \geq r+1$, we see that $y^{\prime} \neq z^{\prime}$. This gives $N(N-2 r)$ equivalence classes.

In group (c), for each word $t$ of length $\leq r$, let $x_{t}$ be the lexicographically least word of length $n-r$ such that $x_{t} t \in C(x)$. (We know such a word exists because each such $t$ is a factor of $x$, considered circularly.) Let $t, u$ be distinct words of length $j$. Then since $\left|x_{t}\right| \geq r+1$, the word $x_{t}$ occurs in exactly one location in $x$, considered circularly, and there it must be followed by $t$. So $x_{t} u \notin C(x)$, so $x_{t}$ and $x_{u}$ are inequivalent under Myhill-Nerode. This gives $v=\left(k^{r+1}-1\right) /(k-1)$ equivalence classes.

Now let us prove the reverse direction. Suppose $x$ is such that $\operatorname{sc}(C(x))=2 v+$ $N(N-2 r-1)+1$. Then from the upper bound in Corollary 1 and the construction of Theorem 3 from which it is derived, we know that all the words corresponding to the states of the automaton in Theorem 3 are pairwise inequivalent under Myhill-Nerode. But there are $k^{r}$ such words of length $r$ and $N$ such words of length $r+1$. Hence, by Proposition 1, we have that $x$ is a generalized de Bruijn word.

For $k=2$ the maximum state complexity of $C(x)$ over length- $N$ words $x$ is given in Table 2 for $1 \leq N \leq 10$. It is sequence A316936 in the OEIS [20]. 


\begin{tabular}{c|c}
$N$ & $\max _{x \in \Sigma_{2}^{N}} \operatorname{sc}(C(x))$ \\
\hline 1 & 3 \\
2 & 5 \\
3 & 7 \\
4 & 11 \\
5 & 15 \\
6 & 21 \\
7 & 29 \\
8 & 39 \\
9 & 49 \\
10 & 61
\end{tabular}

Table 2. Maximum state complexity of conjugates of binary words of length $N$

\section{Final comments}

We do not currently know an accurate asymptotic expression for the number of generalized de Bruijn words of length $N$, except in few simple cases. If $N=k^{n}$, then it follows from known results [1] that this number is (counted up to cyclic shift) $(k !)^{k^{n-1}} / k^{n}$.

Thus far we represented generalized de Bruijn words of length $k^{n}+j$ as closed chains in $G_{k}^{n}$ that visit each vertex. However, in the case of the ordinary de Bruijn word, it is well known that it is more convenient to represent such a word as an Eulerian path in the graph $G_{k}^{n-1}$. This exploits a natural correspondence between edges of $G_{k}^{n-1}$ and vertices of $G_{k}^{n}$. This point of view helps to understand generalized de Bruijn words of length $k^{n}+1$. They correspond to Eulerian paths in $G_{k}^{n-1}$ where one edge is doubled. It is straightforward to see that the only edge which can be doubled so that the resulting graph remains Eulerian is a loop. Therefore, each generalized de Bruijn word of length $k^{n}+1$ is obtained from an ordinary de Bruijn word of length $k^{n}$ by replacing a factor $a^{n-1}$ with $a^{n}$ where $a$ is a single letter. For $k=2$, it follows that the number of such words is $2^{2^{n-1}} / 2^{n-1}$. A similar argument yields the same number of generalized de Bruijn words of length $2^{n}-1$.

Already for $k^{n} \pm 2$ these kinds of considerations become very complex. We leave this as a challenging open problem.

\section{Acknowledgments}

We thank the anonymous referees for helpful comments and suggestions.

\section{References}

1. Aardenne-Ehrenfest, T.v., de Bruijn, N.G.: Circuits and trees in oriented linear graphs. Simon Stevin 28, 203-217 (1951) 
2. Anisiu, M.C., Blázsik, Z., Kása, Z.: Maximal complexity of finite words. Pure Math. Appl. 13, 39-48 (2002)

3. de Bruijn, N.G.: A combinatorial problem. Proc. Konin. Neder. Akad. Wet. 49, 758-764 (1946)

4. de Bruijn, N.G.: Acknowledgement of priority to C. Flye Sainte-Marie on the counting of circular arrangements of $2^{n}$ zeros and ones that show each $n$-letter word exactly once. Tech. Rep. 75-WSK-06, Department of Mathematics and Computing Science, Eindhoven University of Technology, The Netherlands (June 1975)

5. Brzozowski, J.: Quotient complexity of regular languages. J. Automata, Languages, and Combinatorics 15, 71-89 (2010)

6. Etzion, T.: An algorithm for generating shift-register cycles. Theoret. Comput. Sci. 44, 209-224 (1986)

7. Flaxman, A., Harrow, A.W., Sorkin, G.B.: Strings with maximally many distinct subsequences and substrings. Electronic J. Combinatorics 11(1), \#R8 (2004)

8. Flye Sainte-Marie, C.: Question 48. L'Intermédiaire Math. 1, 107-110 (1894)

9. Fredricksen, H.: A survey of full length nonlinear shift register cycle algorithms. SIAM Review 24, 195-221 (1982)

10. Good, I.J.: Normal recurring decimals. J. London Math. Soc. 21, 167-169 (1946)

11. Hemmati, F., Costello, Jr., D.J.: An algebraic construction for $q$-ary shift register sequences. IEEE Trans. Comput. 27, 1192-1195 (1978)

12. Iványi, A.: On the $d$-complexity of words. Ann. Univ. Sci. Budapest. Sect. Comput. 8, 69-90 (1987)

13. Jirásek, J., Jirásková, G.: Cyclic shift on prefix-free languages. In: Bulatov, A.A., Shur, A.M. (eds.) CSR 2013, Lecture Notes in Computer Science, vol. 7913, pp. 246-257. Springer-Verlag (2013)

14. Jirásková, G., Okhotin, A.: State complexity of cyclic shift. RAIRO Inform. Théor. App. 42, 335-360 (2008)

15. Lempel, A.: $m$-ary closed sequences. J. Combin. Theory 10, 253-258 (1971)

16. Martin, M.H.: A problem in arrangements. Bull. Amer. Math. Soc. 40, 859-864 (1934)

17. Maslov, A.N.: Estimates of the number of states of finite automata. Dokl. Akad. Nauk SSSR 194(6), 1266-1268 (1970), in Russian. English translation in Soviet Math. Dokl. 11 (5), 1373-1375 (1970)

18. Ralston, A.: De Bruijn sequences - a model example of the interaction of discrete mathematics and computer science. Math. Mag. 55, 131-143 (1982)

19. Shallit, J.: On the maximum number of distinct factors of a binary string. Graphs and Combinatorics 9, 197-200 (1993)

20. Sloane, N.J.A., et al.: The on-line encyclopedia of integer sequences (2019), available online at https://oeis.org 